\title{
Regularisation and the Mullineux map
}

\author{
Matthew Fayers* \\ Queen Mary, University of London, Mile End Road, London E1 4NS, U.K. \\ m.fayers@qmul.ac.uk \\ Submitted: Aug 12, 2008; Accepted: Nov 14, 2008; Published: Nov 24, 2008 \\ Mathematics Subject Classification: 05E10, 20C30
}

\begin{abstract}
We classify the pairs of conjugate partitions whose regularisations are images of each other under the Mullineux map. This classification proves a conjecture of Lyle, answering a question of Bessenrodt, Olsson and $\mathrm{Xu}$.
\end{abstract}

\section{Introduction}

Suppose $n \geqslant 0$ and $\mathbb{F}$ is a field of characteristic $p$; we adopt the convention that the characteristic of a field is the order of its prime subfield. It is well known that the representation theory of the symmetric group $\Xi_{n}$ is closely related to the combinatorics of partitions. In particular, for each partition $\lambda$ of $n$, there is an important $\mathbb{F}_{n}$-module $S^{\lambda}$ called the Specht module. If $p=\infty$, then the Specht modules are irreducible and afford all irreducible representations of $\mathbb{F}_{n}$. If $p$ is a prime, then for each $p$-regular partition $\lambda$ the Specht module $S^{\lambda}$ has an irreducible cosocle $D^{\lambda}$, and the modules $D^{\lambda}$ afford all irreducible representations of $\mathbb{F S}_{n}$ as $\lambda$ ranges over the set of $p$-regular partitions of $n$.

Given this set-up, it is natural to express representation-theoretic statements in terms of the combinatorics of partitions. An example of this which is of central interest in this paper is the Mullineux map. Let sgn denote the one-dimensional sign representation of $\mathbb{F}_{n}$. Then there is an involutory functor $-\otimes$ sgn from the category of $\mathbb{F}_{n}$-modules to itself. This functor sends simple modules to simple modules, and therefore for each $p$-regular partition $\lambda$ there is some $p$-regular partition $M(\lambda)$ such that $D^{\lambda} \otimes \operatorname{sgn} \cong D^{M(\lambda)}$.

${ }^{*}$ This research was undertaken while the author was visiting Massachusetts Institute.of Technology as a Postdoctoral Fellow, with the support of a Research Fellowship from the Royal Commission for the Exhibition of 1851; the author is very grateful to M.I.T. for its hospitality, and to the 1851 Commission for its generous support. 
The map $M$ thus defined is now called the Mullineux map, since it coincides with a map defined combinatorially by Mullineux [8]; this was proved by Ford and Kleshchev [3], using an alternative combinatorial description of $M$ due to Kleshchev [5].

Another important aspect of the combinatorics of partitions from the point of view of representation theory is $p$-regularisation. This combinatorial procedure was defined by James in order to describe, for each partition $\lambda$, a $p$-regular partition (which is denoted $G \lambda$ in this paper) such that the simple module $D^{G \lambda}$ occurs exactly once as a composition factor of $S^{\lambda}$. In this paper we study the relationship between the Mullineux map and regularisation. Our motivation is the observation that if $p=2$ or $p$ is large relative to the size of $\lambda$, then $M G \lambda=G T \lambda$, where $T \lambda$ denotes the conjugate partition to $\lambda$. However, this is not true for arbitrary $p$, and it natural to ask for which pairs $(p, \lambda)$ we have $M G \lambda=G T \lambda$. The purpose of this paper is to answer this question, which was first posed by Bessenrodt, Olsson and Xu; the answer confirms a conjecture of Lyle.

If we replace the group algebra $\mathbb{F}_{n}$ with the Iwahori-Hecke algebra of the symmetric group at a primitive eth root of unity in $\mathbb{F}$ (for some $e \geqslant 2$ ), then all of the above background holds true, with the prime $p$ replaced by the integer $e$ (and with an appropriate analogue of the sign representation). Therefore, in this paper, we work with an arbitrary integer $e \geqslant 2$ rather than a prime $p$.

In the remainder of this section we give all the definitions we shall need concerning partitions, and state our main result. Section 2 is devoted to proving one half of the conjecture, and Section 3 to the other half. While the first half of the proof consists of elementary combinatorics, the latter half of the proof is algebraic, being an easy consequence of two theorems about $v$-decomposition numbers in the Fock space. We introduce the background material for this as we need it.

\subsection{Partitions}

A partition is a sequence $\lambda=\left(\lambda_{1}, \lambda_{2}, \ldots\right)$ of non-negative integers such that $\lambda_{1} \geqslant$ $\lambda_{2} \geqslant \ldots$ and the sum $|\lambda|=\lambda_{1}+\lambda_{2}+\ldots$ is finite. We say that $\lambda$ is a partition of $|\lambda|$. When writing partitions, we usually group together equal parts and omit zeroes. We write $\varnothing$ for the unique partition of 0 .

$\lambda$ is often identified with its Young diagram, which is the subset

$$
[\lambda]=\left\{(i, j) \mid j \leqslant \lambda_{i}\right\}
$$

of $\mathbb{N}^{2}$. We refer to elements of $\mathbb{N}^{2}$ as nodes, and to elements of $[\lambda]$ as nodes of $\lambda$. We draw the Young diagram as an array of boxes using the English convention, so that $i$ increases down the page and $j$ increases from left to right.

If $e \geqslant 2$ is an integer, we say that $\lambda$ is e-regular if there is no $i \geqslant 1$ such that $\lambda_{i}=\lambda_{i+e-1}>0$, and otherwise we say that $\lambda$ is $e$-singular. We say that $\lambda$ is $e$-restricted if $\lambda_{i}-\lambda_{i+1}<e$ for all $i \geqslant 1$. 


\subsection{Operators on partitions}

Here we introduce a variety of operators on partitions. These include regularisation and the Mullineux map, as well as other more familiar operators which will be useful.

\subsubsection{Conjugation}

Suppose $\lambda$ is a partition. The conjugate partition to $\lambda$ is the partition $T \lambda$ obtained by reflecting the Young diagram along the main diagonal. That is,

$$
(T \lambda)_{i}=\left|\left\{j \geqslant 1 \mid \lambda_{j} \geqslant i\right\}\right| .
$$

We remark that $T \lambda$ is conventionally denoted $\lambda^{\prime}$; we choose our notation in this paper so that all operators on partitions are denoted with capital letters written on the left. The letter $T$ is taken from [1], and stands for 'transpose'.

In this paper we write $l(\lambda)$ for $(T \lambda)_{1}$, i.e. the number of non-zero parts of $\lambda$.

\subsubsection{Row and column removal}

Suppose $\lambda$ is a partition. Let $R \lambda$ denote the partition obtained by removing the first row of the Young diagram; that is, $(R \lambda)_{i}=\lambda_{i+1}$ for $i \geqslant 1$. Similarly, let $C \lambda$ denote the partition obtained by removing the first column from the Young diagram of $\lambda$, i.e. $(C \lambda)_{i}=\max \left\{\lambda_{i}-1,0\right\}$ for $i \geqslant 1$.

In this paper we shall use without comment the obvious relation $T R=C T$.

\subsubsection{Regularisation}

Now we introduce one of the most important concepts of this paper. Suppose $\lambda$ is a partition and $e \geqslant 2$. The $e$-regularisation of $\lambda$ is an $e$-regular partition associated to $\lambda$ in a natural way. The notion of regularisation was introduced by James [4] in the case where $e$ is a prime, where it plays a rôle in the computation of the $e$-modular decomposition matrices of the symmetric groups.

For $l \geqslant 1$, we define the lth ladder in $\mathbb{N}^{2}$ to be the set of nodes $(i, j)$ such that $i+(e-1)(j-1)=l$. The regularisation of $\lambda$ is defined by moving all the nodes of $\lambda$ in each ladder as high as they will go within that ladder. It is a straightforward exercise to show that this procedure gives the Young diagram of a partition, and the $e$-regularisation of $\lambda$ is defined to be this partition.

Example. Suppose $e=3$ and $\lambda=\left(4,3^{3}, 1^{5}\right)$. Then the $e$-regularisation of $\lambda$ is $\left(5,4,3^{2}, 2,1\right)$, as we can see from the following Young diagrams, in which we label each node with 
the number of the ladder in which it lies.

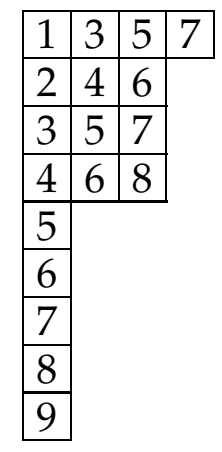

\begin{tabular}{|c|c|c|}
\hline \multicolumn{3}{|c|}{\begin{tabular}{l|l|l|l|l}
1 & 3 & 5 & 7 & 9 \\
\end{tabular}} \\
\hline \multicolumn{3}{|c|}{\begin{tabular}{|l|l|l|l|}
2 & 4 & 6 & 8 \\
\end{tabular}} \\
\hline & \begin{tabular}{|l|l|} 
& 7 \\
\end{tabular} & \\
\hline \begin{tabular}{l|l}
4 & 6 \\
\end{tabular} & \begin{tabular}{|l|l|} 
\\
\end{tabular} & \\
\hline & & \\
\hline 4 & & \\
\hline
\end{tabular}

We write $G \lambda$ for the $e$-regularisation of $\lambda$. Clearly $G \lambda$ is $e$-regular, and equals $\lambda$ if $\lambda$ is $e$-regular. We record here three results we shall need later; the proofs of the first two are easy exercises.

Lemma 1.1. Suppose $\lambda$ is a partition. If $(G \lambda)_{1}=\lambda_{1}$, then $R G \lambda=G R \lambda$.

Lemma 1.2. Suppose $\lambda$ and $\mu$ are partitions. If $l(\lambda)=l(\mu)$ and $G C \lambda=C \mu$, then $G \lambda=G \mu$.

Lemma 1.3. Suppose $\zeta$ is an e-regular partition, and $x \geqslant l(\zeta)+e-1$. Let $\xi$ be the partition obtained by adding a column of length $x$ to $\zeta$, and let $\eta$ be the partition obtained by adding $a$ column of length $x-e+1$ to $C \zeta$. Then $G \eta=C G \xi$.

Proof. For any $n \geqslant 1$ and any partition $\lambda$, let $\operatorname{lad}_{n}(\lambda)$ denote the number of nodes of $\lambda$ in ladder $n$. Since $G \eta$ and $C G \xi$ are both $e$-regular, it suffices to show that $\operatorname{lad}_{n}(G \eta)=$ $\operatorname{lad}_{n}(C G \xi)$ for all $n$.

$\eta$ is obtained from $\zeta$ by adding the nodes $(l(\zeta)+1,1), \ldots,(x-e+1,1)$, so we have

$$
\operatorname{lad}_{n}(G \eta)=\operatorname{lad}_{n}(\eta)= \begin{cases}\operatorname{lad}_{n}(\zeta)+1 & (l(\zeta)<n<x+e) \\ \operatorname{lad}_{n}(\zeta) & \text { (otherwise). }\end{cases}
$$

It is also easy to compute

$$
\operatorname{lad}_{n}(\xi)= \begin{cases}1 & (1 \leqslant n<e) \\ \operatorname{lad}_{n-e+1}(\zeta)+1 & (e \leqslant n \leqslant x) \\ \operatorname{lad}_{n-e+1}(\zeta) & (x<n)\end{cases}
$$

Claim. $l(G \xi)=l(\zeta)+e-1$.

Proof. Since $\zeta$ is $e$-regular and $(l(\zeta), 1) \in[\zeta]$, every node of ladder $l(\zeta)$ is a node of $\zeta$. Hence every node of ladder $l(\zeta)+e-1$ is a node of $\xi$; so when $\xi$ is regularised, none of these nodes moves, and we have $(l(\zeta)+e-1,1) \in[G \xi]$, i.e. $l(G \xi) \geqslant l(\zeta)+e-1$.

On the other hand, the node $(l(\zeta)+1,2)$ does not lie in $[\xi]$, so the node $(l(\zeta)+e, 1)$ cannot lie in $[G \xi]$, i.e. $l(G \xi)<l(\zeta)+e$. 
From the claim we deduce that

$$
\operatorname{lad}_{n}(C G \xi)= \begin{cases}\operatorname{lad}_{n+e-1}(\xi)-1 & (n \leqslant l(\zeta)) \\ \operatorname{lad}_{n+e-1}(\xi) & (n>l(\zeta)),\end{cases}
$$

and combining this with the statements above gives the result.

\subsubsection{The Mullineux map}

Now we introduce the Mullineux map, which is the most important concept of this paper. We shall give two different recursive definitions of the Mullineux map: the original definition due to Mullineux [8], and an alternative version due to $\mathrm{Xu}$ [9].

Suppose $\lambda$ is a partition, and define the rim of $\lambda$ to be the subset of $[\lambda]$ consisting of all nodes $(i, j)$ such that $(i+1, j+1) \notin \lambda$. Now fix $e \geqslant 2$, and suppose that $\lambda$ is $e$-regular. Define the $e$-rim of $\lambda$ to be the subset $\left\{\left(i_{1}, j_{1}\right), \ldots,\left(i_{r}, j_{r}\right)\right\}$ of the rim of $\lambda$ obtained by the following procedure.

- If $\lambda=\varnothing$, then set $r=0$, so that the $e$-rim of $\lambda$ is empty. Otherwise, let $\left(i_{1}, j_{1}\right)$ be the top-rightmost node of the rim, i.e. the node $\left(1, \lambda_{1}\right)$.

- For $k>1$ with $e \nmid k-1$, let $\left(i_{k}, j_{k}\right)$ be the next node along the rim from $\left(i_{k-1}, j_{k-1}\right)$, i.e. the node $\left(i_{k-1}+1, j_{k-1}\right)$ if $\lambda_{i_{k-1}}=\lambda_{i_{k-1}+1}$, or the node $\left(i_{k-1}, j_{k-1}-1\right)$ otherwise.

- For $k>1$ with $e \mid k-1$, define $\left(i_{k}, j_{k}\right)$ to be the node $\left(i_{k-1}+1, \lambda_{i_{k-1}+1}\right)$.

- Continue until a node $\left(i_{k}, j_{k}\right)$ is reached in the bottom row of $[\lambda]$ (i.e. with $i_{k}=l(\lambda)$ ), and either $j_{k}=1$ or $e \mid k$. Set $r=k$, and stop.

Less formally, we construct the $e$-rim of $\lambda$ by working along the rim from top right to bottom left, and moving down one row every time the number of nodes we've seen is divisible by $e$.

The integer $r$ defined in this way is called the e-rim length of $\lambda$. We define $I \lambda$ to be the partition obtained by removing the $e$-rim of $\lambda$ from $[\lambda]$.

\section{Examples.}

1. Suppose $e=3$, and $\lambda=\left(10,6^{2}, 4,2\right)$. Then the $e$-rim of $\lambda$ consists of the marked nodes in the following diagram, and we see that $r=11$ and $I \lambda=(7,5,4,1)$.

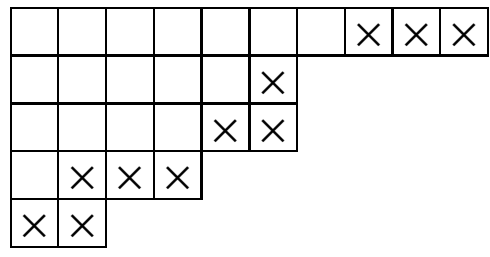


2. Suppose $e=2$, and $\lambda$ is any 2-regular partition. The 2-rim of $\lambda$ consists of the last two nodes in each row of $[\lambda]$ (or the last node, if there is only one). Hence when $e=2$ the operator $I$ is the same as $C^{2}$.

Now we can define the Mullineux map recursively. Suppose $\lambda$ is an $e$-regular partition. If $\lambda=\varnothing$, then set $M \lambda=\varnothing$. Otherwise, compute the partition $I \lambda$ as above. Then $|I \lambda|<|\lambda|$, and $I \lambda$ is $e$-regular, so we may assume that MI $\lambda$ is defined. Let $r$ be the $e$-rim length of $\lambda$, and define

$$
m= \begin{cases}r-l(\lambda) & (e \mid r) \\ r-l(\lambda)+1 & (e \nmid r) .\end{cases}
$$

It turns out that there is a unique $e$-regular partition $\mu$ which has $e$-rim length $r$ and $l(\mu)=m$, and which satisfies $I \mu=M I \lambda$. We set $M \lambda=\mu$.

\section{Examples.}

1. Suppose $e=3, \lambda=\left(3^{2}, 2^{2}, 1\right)$ and $\mu=(6,4,1)$. Then we have $I \lambda=\left(2,1^{2}\right)$ and $I \mu=(3,1)$, as we see from the following diagrams.
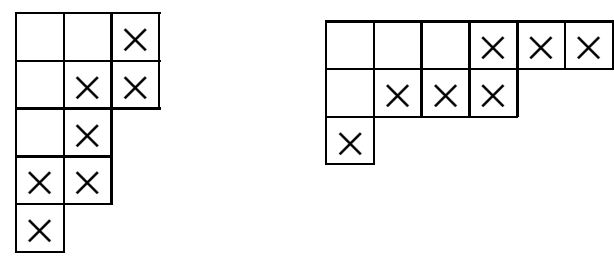

Computing $e$-rims again, we find that $I^{2} \lambda=I^{2} \mu=\varnothing$. Now comparing the numbers of non-zero parts of these partitions with their $e$-rim lengths we find that $M I \lambda=I \mu$, and hence that $M \lambda=\mu$.

2. Suppose $e=2$, and $\lambda$ is a 2-regular partition. From above, we see that the 2rim length of $\lambda$ is $2 l(\lambda)$, if $\lambda_{l(\lambda)} \geqslant 2$, or $2 l(\lambda)-1$ if $\lambda_{l(\lambda)}=1$. Either way, we get $m=l(\lambda)$, and this implies inductively that in the case $e=2$ the Mullineux map is the identity.

3. Suppose $e$ is large relative to $\lambda$; in particular, suppose $e$ is greater than the number of nodes in the rim of $\lambda$. Then the $e$-rim of $\lambda$ coincides with the rim, so that the $e$-rim length is $\lambda_{1}+l(\lambda)-1$. Hence $m=\lambda_{1}$, and from this it is easy to prove by induction that $M \lambda=T \lambda$.

Now we give Xu's alternative definition of the Mullineux map. Suppose $\lambda$ is a partition with $e$-rim length $r$, and define

$$
l^{\prime}= \begin{cases}l(\lambda) & (e \mid r) \\ l(\lambda)-1 & (e \nmid r) .\end{cases}
$$


Define $J \lambda$ to be the partition obtained by removing the $e$-rim from $\lambda$, and then adding a column of length $l^{\prime}$. Another way to think of this is to define the truncated e-rim of $\lambda$ to be the set of nodes $(i, j)$ in the $e$-rim of $\lambda$ such that $(i, j-1)$ also lies in the $e$-rim, together with the node $(l(\lambda), 1)$ if $e \nmid r$, and to define $J \lambda$ to be the partition obtained by removing the truncated $e$-rim.

Example. Returning to an earlier example, take $e=3$ and $\lambda=\left(10,6^{2}, 4,2\right)$. Then the truncated $e$-rim of $\lambda$ consists of the marked nodes in the following diagram, and we see that $I \lambda=(8,6,5,2)$.

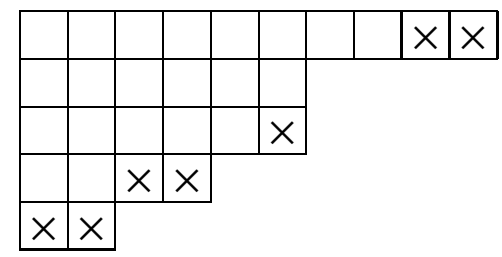

If $\lambda$ is $e$-regular, then it is a simple exercise to show that $J \lambda$ is $e$-regular and $|J \lambda|<|\lambda|$. So we assume that $M J \lambda$ is defined recursively, and we define $M \lambda$ to be the partition obtained by adding a column of length $|\lambda|-|J \lambda|$ to $M J \lambda$. Xu [9, Theorem 1] shows that this map coincides with Mullineux's map $M$. In other words, we have the following.

Proposition 1.4. Suppose $\lambda$ and $\mu$ are e-regular partitions, with $|\lambda|=|\mu|$. Then $M \lambda=\mu$ if and only if $M J \lambda=C \mu$.

\subsection{Hooks}

Now we set up some basic notation concerning hooks in Young diagrams. Suppose $\lambda$ is a partition, and $(i, j)$ is a node of $\lambda$. The $(i, j)$-hook of $\lambda$ is defined to be the set $H_{i j}(\lambda)$ of nodes in $[\lambda]$ directly to the right of or directly below $(i, j)$, including the node $(i, j)$ itself. The $\operatorname{arm}$ length $a_{i j}(\lambda)$ is the number of nodes directly to the right of $(i, j)$, i.e. $\lambda_{i}-j$, and the leg length $l_{i j}(\lambda)$ is the number of nodes directly below $(i, j)$, i.e. $(T \lambda)_{j}-i$. The $(i, j)$-hook length $h_{i j}(\lambda)$ is the total number of nodes in $H_{i j}(\lambda)$, i.e. $a_{i j}(\lambda)+l_{i j}(\lambda)+1$.

Now fix $e \geqslant 2$. The $e$-weight of $\lambda$ is defined to be the number of nodes $(i, j)$ of $\lambda$ such that $e \mid h_{i j}(\lambda)$. If $(i, j) \in[\lambda]$ with $e \mid h_{i j}(\lambda)$, we say that $H_{i j}(\lambda)$ is

- shallow if $a_{i j}(\lambda) \geqslant(e-1) l_{i j}(\lambda)$, or

- steep if $l_{i j}(\lambda) \geqslant(e-1) a_{i j}(\lambda)$.

Example. Suppose $e=3$ and $\lambda=\left(5,2,1^{4}\right)$. Then we have $(2,1) \in[\lambda]$, with $a_{2,1}(\lambda)=1$, $l_{2,1}(\lambda)=4$, and hence $h_{2,1}(\lambda)=6 . H_{2,1}(\lambda)$ is steep if $e=3$, but not if $e=6$.

\subsection{Lyle's Conjecture}

Suppose $e \geqslant 2$ and $\lambda$ is an $e$-regular partition. As noted above, if $e$ is large relative to $|\lambda|$, then $M \lambda=T \lambda$. Of course, there is no hope that this is true in general, since $T \lambda$ will 
not in general be an $e$-regular partition. But $e$-regularisation provides a natural way to obtain an $e$-regular partition from an arbitrary partition, and it is therefore natural to ask: for which $e$-regular partitions $\lambda$ do we have $M \lambda=G T \lambda$ ? When $e$ is large relative to $\lambda$ we have $G \lambda=\lambda$ and (from the example above) $M \lambda=T \lambda$, so certainly $M \lambda=G T \lambda$ in this case. We also have $M \lambda=G T \lambda$ for all partitions $\lambda$ when $e=2$ : we have seen that for $e=2$ the Mullineux map is the identity, and it is a simple exercise to show that $\lambda$ and $T \lambda$ have the same 2-regularisation for any $\lambda$. But it is not generally true that $M \lambda=G T \lambda$ for an $e$-regular partition $\lambda$. Bessenrodt, Olsson and $\mathrm{Xu}$ [1] have given a classification of the partitions for which this does hold, as follows.

Theorem 1.5. [1, Theorem 4.8] Suppose $\lambda$ is an e-regular partition. Then $M \lambda=G T \lambda$ if and only if for every $(i, j) \in[\lambda]$ with $e \mid h_{i j}(\lambda)$, the hook $H_{i j}(\lambda)$ is shallow.

Example. Suppose $e=4$ and $\lambda=\left(14,10,2^{2}\right)$. The Young diagram is as follows; we have marked those nodes $(i, j)$ for which $4 \mid h_{i j}(\lambda)$.

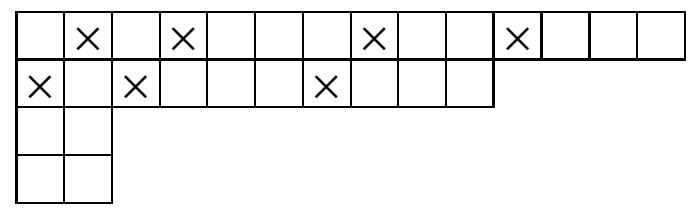

We see that all the hooks of length divisible by 4 are shallow, so $\lambda$ satisfies the second hypothesis of Theorem 1.5. And it may be verified that $G T \lambda=M \lambda=\left(5^{2}, 4^{2}, 3^{2}, 2^{2}\right)$.

Bessenrodt, Olsson and $\mathrm{Xu}$ have also posed the following more general question [1, p. 454], which is essentially the same problem without the assumption that $\lambda$ is e-regular.

For which partitions $\lambda$ is it true that $M G \lambda=G T \lambda$ ?

Motivated by the (now solved) problem of the classification of irreducible Specht modules for symmetric groups, Lyle conjectured the following solution in her thesis.

Conjecture 1.6. [7, Conjecture 5.1.18] Suppose $\lambda$ is a partition. Then $M G \lambda=G T \lambda$ if and only if for every $(i, j) \in[\lambda]$ with $e \mid h_{i j}(\lambda)$, the hook $H_{i j}(\lambda)$ is either shallow or steep.

The purpose of this paper is to prove this conjecture. It is a simple exercise to show that a partition possessing a steep hook must be $e$-singular; so in the case where $\lambda$ is $e$-regular, Conjecture 1.6 reduces to Theorem 1.5.

Let us define an L-partition to be a partition satisfying the second condition of Conjecture 1.6, i.e. a partition for which every $H_{i j}(\lambda)$ of length divisible by $e$ is either shallow or steep. 
Example. Suppose $e=4$ and $\lambda=\left(11,2^{2}, 1^{5}\right)$. The Young diagram of $\lambda$ is as follows.

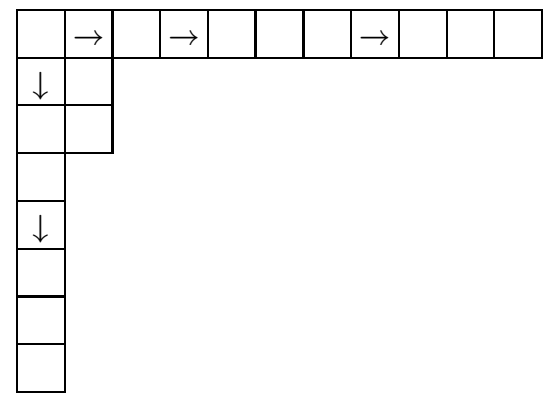

The nodes $(i, j)$ with $4 \mid h_{i j}(\lambda)$ are marked; we see that those marked $\rightarrow$ correspond to shallow hooks, and those marked $\downarrow$ correspond to steep hooks. So $\lambda$ is an L-partition when $e=4$. We have $G \lambda=\left(11,3,2^{2}, 1^{2}\right), G T \lambda=\left(8,4,3^{2}, 2\right)$, and it can be checked that $M G \lambda=G T \lambda$.

\section{The 'if' part of Conjecture $\mathbf{1 . 6}$}

In this section we prove the 'if' half of Conjecture 1.6, i.e. that $M G \lambda=G T \lambda$ whenever $\lambda$ is an L-partition. We begin by noting some properties of L-partitions, and making some more definitions. Note that when $e=2$, every partition is an L-partition; by the above remarks we have $M G \lambda=G T \lambda$ for every partition when $e=2$, so Conjecture 1.6 holds when $e=2$. Therefore, we assume throughout this section that $e \geqslant 3$. The following simple observations will be used without comment.

Lemma 2.1. Suppose $\lambda$ is a partition. Then $\lambda$ is an L-partition if and only if $T \lambda$ is. If $\lambda$ is an $L$-partition, then so are $R \lambda$ and $C \lambda$.

Now we examine the structure of L-partitions in more detail. Suppose $\lambda$ is an Lpartition, and let $s(\lambda)$ be maximal such that $\lambda_{s(\lambda)}-\lambda_{s(\lambda)+1} \geqslant e$, setting $s(\lambda)=0$ if $\lambda$ is $e$-restricted. Similarly, set $t(\lambda)=0$ if $\lambda$ is $e$-regular, and otherwise let $t(\lambda)$ be maximal such that $(T \lambda)_{t(\lambda)}-(T \lambda)_{t(\lambda)+1} \geqslant e$. Clearly, we have $s(\lambda)=t(T \lambda)$.

Lemma 2.2. If $\lambda$ is an L-partition, then for $1 \leqslant i \leqslant s(\lambda)$ we have $\lambda_{i}-\lambda_{i+1} \geqslant e-1$, while for $1 \leqslant j \leqslant t(\lambda)$ we have $(T \lambda)_{j}-(T \lambda)_{j+1} \geqslant e-1$.

Proof. We prove the first statement. Suppose this statement is false, and let $i<s(\lambda)$ be maximal such that $\lambda_{i}-\lambda_{i+1}<e-1$. Put $j=\lambda_{i}-e+2$. Then we have $(i, j) \in[\lambda]$, with $a_{i j}(\lambda)=e-2$ and $l_{i j}(\lambda)=1$, which (given our assumption that $e \geqslant 3$ ) contradicts the assumption that $\lambda$ is an L-partition.

Lemma 2.3. Suppose $\lambda$ is an L-partition and $(i, j) \in[\lambda]$ with $e \mid h_{i j}(\lambda)$.

1. If $i>s(\lambda)$, then $H_{i j}(\lambda)$ is steep. 
2. If $j>t(\lambda)$, then $H_{i j}(\lambda)$ is shallow.

Proof. We prove (1). Let $a=a_{i j}(\lambda)$ and $l=l_{i j}(\lambda) . \lambda$ is an L-partition, so if $H_{i j}(\lambda)$ is not steep then it must be shallow, i.e. $a \geqslant(e-1) l$. In fact, since $e \mid h_{i j}(\lambda)=a+l+1$, we find that $a \geqslant(e-1) l+e-1$. The definition of $l$ implies that $\lambda_{i+l+1}<j=\lambda_{i}-a$, so

$$
\lambda_{i}-\lambda_{i+l+1}>a \geqslant(e-1)(l+1),
$$

which implies that for some $k \in\{i, \ldots, i+l\}$ we have $\lambda_{k}-\lambda_{k+1} \geqslant e$. But this contradicts the assumption that $i>s(\lambda)$.

Now we define an operator $S$ on L-partitions. Suppose $\lambda$ is an L-partition, and let $s=s(\lambda)$. Define

$$
S \lambda=\left(\lambda_{1}-e+1, \lambda_{2}-e+1, \ldots, \lambda_{s}-e+1, \lambda_{s+2}, \lambda_{s+3}, \ldots\right) .
$$

Note that if $\lambda$ is an $e$-restricted L-partition, then $S \lambda=R \lambda$. In general, we need to know that $S$ maps L-partitions to L-partitions, in order to allow an inductive proof of Conjecture 1.6.

Lemma 2.4. If $\lambda$ is an L-partition, then so is $S \lambda$.

Proof. Suppose $\lambda$ is an L-partition, and that $(i, j) \in[S \lambda]$.

If $i>s(\lambda)$, then $(i+1, j) \in[\lambda]$, and we have

$$
a_{i j}(S \lambda)=a_{(i+1) j}(\lambda), \quad l_{i j}(S \lambda)=l_{(i+1) j}(\lambda) .
$$

So if $e \mid h_{i j}(S \lambda)$, then $e \mid h_{(i+1) j}(\lambda)$; so by Lemma 2.3(1) $H_{(i+1) j}(\lambda)$ is steep, and therefore $H_{i j}(S \lambda)$ is steep.

Next suppose $i \leqslant s(\lambda)$ and $j>\lambda_{s+1}$. Then $(i, j+e-1) \in[\lambda]$ and $a_{i j}(S \lambda)=a_{i(j+e-1)}(\lambda)$, $l_{i j}(S \lambda)=l_{i(j+e-1)}(\lambda)$. So if $e \mid h_{i j}(S \lambda)$, then $e \mid h_{i(j+e-1)}(\lambda)$, and so $H_{i(j+e-1)}(\lambda)$ is shallow, and hence $H_{i j}(S \lambda)$ is shallow.

Finally, suppose that $i \leqslant s(\lambda)$ and $j \leqslant \lambda_{s+1}$. Then $(i, j) \in[\lambda]$, and we have

$$
a_{i j}(S \lambda)=a_{i j}(\lambda)-e+1, \quad l_{i j}(S \lambda)=l_{i j}(\lambda)-1 .
$$

So if $e \mid h_{i j}(S \lambda)$, then $e \mid h_{i j}(\lambda)$, and hence $H_{i j}(\lambda)$ is either shallow or steep. If it is shallow, then we have

$$
a_{i j}(S \lambda)=a_{i j}(\lambda)-e+1 \geqslant(e-1) l_{i j}(\lambda)-e+1=(e-1) l_{i j}(S \lambda),
$$

so that $H_{i j}(S \lambda)$ is shallow. On the other hand, if $H_{i j}(\lambda)$ is steep, then

$$
l_{i j}(S \lambda)=l_{i j}(\lambda)-1 \geqslant(e-1) a_{i j}(\lambda)-1>(e-1) a_{i j}(S \lambda)
$$

so $H_{i j}(S \lambda)$ is steep. 
Example. Suppose $e=3$, and let $\lambda=\left(9,5,2,1^{5}\right)$. Then we have $s(\lambda)=2$, so that $S \lambda=\left(7,3,1^{5}\right)$. We see that both $\lambda$ and $S \lambda$ are L-partitions from the following diagrams.
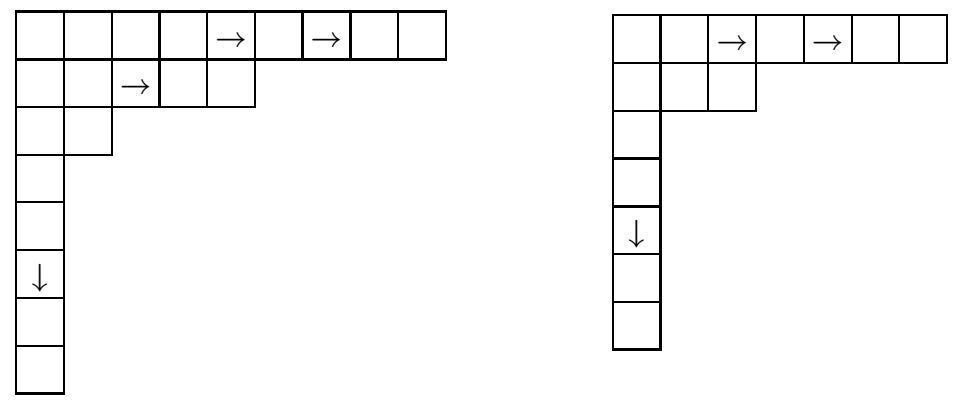

Now we examine the relationship between the operator $S$ and $e$-regularisation.

Lemma 2.5. Suppose $\lambda$ is an L-partition. Then

$$
G T S \lambda=C G T \lambda .
$$

Proof. We use induction on $s(\lambda)$. In the case $s(\lambda)=0$ both $\lambda$ and $S \lambda=R \lambda$ are $e$-restricted, i.e. $T \lambda$ and $T S \lambda$ are $e$-regular, and so GTS $\lambda=T S \lambda=T R \lambda=C T \lambda=C G T \lambda$.

Now suppose $s(\lambda)>0$. Then $s(R \lambda)=s(\lambda)-1$, so we may assume that the result holds with $\lambda$ replaced by $R \lambda$. Put $\zeta=G C T \lambda$; then by the inductive hypothesis GTSR $\lambda=$ CGTR $\lambda=C \zeta$. Let $\xi$ and $\eta$ be as defined in Lemma 1.3, with $x=\lambda_{1}$. Note that

$$
x=\lambda_{1} \geqslant \lambda_{2}+e-1=l(C T \lambda)+e-1 \geqslant l(G C T \lambda)+e-1=l(\zeta)+e-1,
$$

as required by Lemma 1.3.

Claim. $G T \lambda=G \xi$.

Proof. We have $l(T \lambda)=\lambda_{1}=l(\xi)$ and GCT $\lambda=\zeta=C \xi$, and Lemma 1.2 gives the result.

Claim. GTS $\lambda=G \eta$.

Proof. Since $s(\lambda)>0, S \lambda$ may be obtained from $S R \lambda$ by adding a row of length $\lambda_{1}-e+1$; hence TS $\lambda$ may be obtained from TSR $\lambda$ by adding a column of length $\lambda_{1}-e+1$. So we have $l(T S \lambda)=\lambda_{1}-e+1=l(\eta)$, and

$$
\text { GCTS } \lambda=G T S R \lambda=C \zeta=C \eta,
$$

and again we may appeal to Lemma 1.2.

Now Lemma 1.3 combined with these two claims gives the result.

Next we prove a simple lemma which gives an equivalent statement to the condition $M G \lambda=G T \lambda$ in the presence of a suitable inductive hypothesis. 
Lemma 2.6. Suppose $\lambda$ is an L-partition, and that $M G \mu=G T \mu$ for all L-partitions $\mu$ with $|\mu|<|\lambda|$. Then $M G \lambda=G T \lambda$ if and only if $G S \lambda=J G \lambda$.

Proof. Since $|G \lambda|=|G T \lambda|$, we have

$$
\begin{array}{rlr}
M G \lambda=G T \lambda & \Longleftrightarrow M J G \lambda=C G T \lambda & \text { by Proposition } 1.4 \\
& \Longleftrightarrow M J G \lambda=G T S \lambda & \text { by Lemma 2.5 } \\
& \Longleftrightarrow M J G \lambda=M G S \lambda & \text { by the inductive hypothesis and Lemma 2.4 } \\
& \Longleftrightarrow J G \lambda=G S \lambda .
\end{array}
$$

We now require one more lemma concerning the regularisations of L-partitions.

Lemma 2.7. Suppose $\lambda$ is an L-partition with $s(\lambda)>0$ and $\lambda_{1} \geqslant l(\lambda)$. Then:

1. $(G \lambda)_{1}=\lambda_{1}$;

2. $(G \lambda)_{1}-(G \lambda)_{2} \geqslant e-1$;

3. $(G S \lambda)_{1}=(S \lambda)_{1}$.

\section{Proof.}

1. Obviously $(G \lambda)_{1} \geqslant \lambda_{1}$, so it suffices to show that $[\lambda]$ does not contain a node in ladder $(e-1) \lambda_{1}+1$. If it does, let $(i, j)$ be the rightmost such node. Since $(i, j) \neq\left(1, \lambda_{1}+1\right)$, we have $i \geqslant e$ and we know that the node $(i-e+1, j+1)$ does not lie in $\lambda$; in other words, $(T \lambda)_{j}-(T \lambda)_{j+1} \geqslant e$. This means that $j \leqslant t(\lambda)$, and so by Lemma 2.2 we have $i \leqslant l(\lambda)-(e-1)(j-1)$, so that

$$
l(\lambda) \geqslant i+(e-1)(j-1)=(e-1) \lambda_{1}+1>\lambda_{1},
$$

contrary to hypothesis.

2. By part (1), we must show that $(G \lambda)_{2} \leqslant \lambda_{1}-e+1$, i.e. that $[\lambda]$ does not contain a node in ladder $2+(e-1)\left(\lambda_{1}-e+1\right)$. Supposing otherwise, we let $(i, j)$ be the rightmost such node. Arguing as above, we find that

$$
\lambda_{1} \geqslant l(\lambda) \geqslant i+(e-1)(j-1)=2+(e-1)\left(\lambda_{1}-e+1\right),
$$

and this rearranges to yield $\lambda_{1}<e$, which is absurd given that $s(\lambda)>0$.

3. Obviously $(G S \lambda)_{1} \geqslant(S \lambda)_{1}=\lambda_{1}-e+1$, so it suffices to show that $[S \lambda]$ does not contain a node in ladder $1+(e-1)\left(\lambda_{1}-e+1\right)$. Arguing as above, such a node would have to be of the form $(i, j)$ with $j \leqslant t(S \lambda) \leqslant t(\lambda)$. But then $(T S \lambda)_{j}=(T \lambda)_{j}-1$, so $[\lambda]$ contains the node $(i+1, j)$, which lies in ladder $2+(e-1)\left(\lambda_{1}-e+1\right)$. But it was shown in (2) that this is not possible. 
Proof of Conjecture 1.6 ('if' part). We proceed by induction on $|\lambda|$. It is clear that $\lambda$ is an L-partition if and only if $T \lambda$ is, so Conjecture 1.6 holds for $\lambda$ if and only if it holds for $T \lambda$. If either $\lambda$ or $T \lambda$ is $e$-regular, then the result follows from Theorem 1.5, so we assume that $\lambda$ is neither $e$-regular nor $e$-restricted; in particular, $s(\lambda)>0$. By replacing $\lambda$ with $T \lambda$ if necessary, we assume also that $\lambda_{1} \geqslant l(\lambda)$.

Claim. $(J G \lambda)_{1}=\lambda_{1}-e+1$, and $R J G \lambda=J G R \lambda$.

Proof. This follows from Lemma 2.7(1-2), given the definition of the operator $J$.

Claim. $(G S \lambda)_{1}=\lambda_{1}-e+1$, and $R G S \lambda=G R S \lambda$.

Proof. We have $(S \lambda)_{1}=\lambda_{1}-e+1$ by definition, and $(G S \lambda)_{1}=(S \lambda)_{1}$ by Lemma 2.7(3). The second statement follows from Lemma 1.1.

By induction (replacing $\lambda$ with $R \lambda$ ) we have $M G R \lambda=G T R \lambda$, and by Lemma 2.6 (and the inductive hypothesis) this gives $J G R \lambda=G S R \lambda$. Since obviously GSR $\lambda=G R S \lambda$, the two claims yield $J G \lambda=G S \lambda$. Now applying Lemma 2.6 again gives the result.

\section{The Fock space and v-decomposition numbers}

In this section, we complete the proof of Conjecture 1.6 using $v$-decomposition numbers. We give only a very brief sketch of the background material needed, since this is discussed at length elsewhere; in particular, the article of Lascoux, Leclerc and Thibon [6] is an invaluable source.

Fix $e \geqslant 2$, let $v$ be an indeterminate over $\mathbb{Q}$, and let $\mathcal{U}$ be the quantum algebra $U_{v}\left(\widehat{\mathfrak{s l}}_{e}\right)$ over $\mathbb{Q}(v)$. There is a module $\mathcal{F}$ for this algebra called the Fock space, which has a standard basis indexed by (and often identified with) the set of all partitions. The submodule generated by the empty partition is isomorphic to the basic representation of $\mathcal{U}$. This submodule has a canonical $\mathbb{Q}(v)$-basis

$$
\{G(\mu) \mid \mu \text { an } e \text {-regular partition }\} \text {. }
$$

The v-decomposition numbers are the coefficients obtained when the elements of the canonical basis are expanded in terms of the standard basis, i.e. the coefficients $d_{\lambda \mu}(v)$ in the expression

$$
G(\mu)=\sum_{\lambda} d_{\lambda \mu}(v) \lambda
$$

We shall need to quote two results concerning v-decomposition numbers; one concerning the Mullineux map, and the other concerning $e$-regularisation. The first of these involves the $e$-weight of a partition, defined in $§ 1.3$. 
Theorem 3.1. [6, Theorem 7.2] Suppose $\lambda$ and $\mu$ are partitions with e-weight $w$, and that $\mu$ is e-regular. Then

$$
d_{(T \lambda)(M \mu)}(v)=v^{w} d_{\lambda \mu}\left(v^{-1}\right) .
$$

The second result we need requires a definition. Given a partition $\lambda$, let $z(\lambda)$ be the number of nodes $(i, j) \in[\lambda]$ such that $e \mid h_{i j}(\lambda)$ and $H_{i j}(\lambda)$ is steep. Now we have the following result.

Theorem 3.2. [2, Theorem 2.2] For any partition $\lambda$,

$$
d_{\lambda(G \lambda)}(v)=v^{z(\lambda)} .
$$

Remark. Note that in [2] an alternative convention for the Fock space is used: our $d_{\lambda \mu}(v)$ is written in [2] as $d_{(T \lambda)(T \mu)}(v)$. Accordingly, the statement of [2, Theorem 2.2] involves shallow hooks rather than steep hooks. We hope that no confusion will result.

Now we combine these theorems. First we note the following obvious result about $e$-weight and the function $z$.

Lemma 3.3. Suppose $\lambda$ is a partition with e-weight $w$. Then $T \lambda$ also has e-weight $w$, and $z(T \lambda)$ equals the number of nodes $(i, j) \in[\lambda]$ such that $e \mid h_{i j}(\lambda)$ and $H_{i j}(\lambda)$ is shallow. Hence $\lambda$ is an L-partition if and only if $w=z(\lambda)+z(T \lambda)$.

Now we can complete the proof of Conjecture 1.6.

Proof of Conjecture 1.6 ('only if' part). Suppose $M G \lambda=G T \lambda$, and that $\lambda$ has $e$-weight $w$. Then we have

$$
\begin{aligned}
v^{z(T \lambda)} & =d_{(T \lambda)(G T \lambda)}(v) \\
& =d_{(T \lambda)(M G \lambda)}(v) \\
& =v^{w} d_{\lambda(G \lambda)}\left(v^{-1}\right) \\
& =v^{w} \cdot v^{-z(\lambda)}
\end{aligned}
$$

by Theorem 3.2

by hypothesis

by Theorem 3.1

by Theorem 3.2

so that $w=z(\lambda)+z(T \lambda)$. Now Lemma 3.3 gives the result.

\section{References}

[1] C. Bessenrodt, J. Olsson \& M. Xu, 'On properties of the Mullineux map with an application to Schur modules', Math. Proc. Cambridge Philos. Soc. 126 (1999), 443-59.

[2] M. Fayers, ' $q$-analogues of regularisation theorems for linear and projective representations of the symmetric group', J. Algebra 316 (2007), 346-67.

[3] B. Ford \& A. Kleshchev, 'A proof of the Mullineux conjecture', Math. Z. 226 (1997), 267-308. 
[4] G. James, 'On the decomposition matrices of the symmetric groups II', J. Algebra 43 (1976), 45-54.

[5] A. Kleshchev, 'Branching rules for modular representations of symmetric groups, III: some corollaries and a problem of Mullineux', J. London Math. Soc. (2) 54 (1996), 25-38.

[6] A. Lascoux, B. Leclerc \& J.-Y. Thibon, 'Hecke algebras at roots of unity and crystal bases of quantum affine algebras', Comm. Math. Phys. 181 (1996), 205-63.

[7] S. Lyle, Some topics in the representation theory of the symmetric and general linear groups, Ph.D. thesis, University of London, 2003.

[8] G. Mullineux, 'Bijections on $p$-regular partitions and $p$-modular irreducibles of the symmetric groups', J. London Math. Soc. (2) 20 (1979), 60-6.

[9] M. Xu, 'On Mullineux's conjecture in the representation theory of symmetric groups', Comm. Alg. 25 (1997), 1797-803. 